\section{Abnormal Cardiac Biomarkers in Patients with Systemic Lupus Erythematosus and No Prior Heart Disease: The Role of Endomyocardial Biopsy}

\section{To the Editor:}

Tselios, et al reported in their recent study that patients with systemic lupus erythematosus (SLE) who receive prolonged antimalarial (AM) treatment are at increased risk for elevated cardiac biomarkers [brain natriuretic peptide (BNP) and high-sensitivity cardiac troponin I (HS-cTnI)], particularly when persistently elevated creatine phosphokinase (CPK) is present ${ }^{1}$. Among a well-characterized SLE cohort of 179 patients, the authors found that 16 (all without cardiac disease, renal failure, or pulmonary arterial hypertension) presented an increase of at least 1 cardiac biomarker. Prolonged AM use (> 5.6 yrs) was found to be associated with these laboratory abnormalities, regardless of age and SLE disease duration ${ }^{1}$. Regarding the potential reason underlying these findings, the authors speculated that AM deposition in muscle tissue (expressed with chronic CPK elevation) could not be restricted to skeletal muscles, and the drug could also accumulate within the myocardium. Therefore, they proposed the evaluation of these cardiac biomarkers as a screening tool for patients with SLE receiving AM treatment for longer than 5.6 years and/or with persistently elevated CK levels.

The authors' findings open a new and interesting scenario about the prevalence and causes of AM-induced cardiotoxicity in patients with SLE. Their conclusions, though, might deserve a deeper discussion before being applied to clinical practice, especially for concerning the pathogenic mechanisms leading to cardiac biomarker increases in systemic inflammatory diseases such as SLE, and the diagnostic and prognostic value of endomyocardial biopsy (EBM) in this clinical scenario. It is indeed known that in this clinical setting, raised HS-cTnI serum levels might be due to the increased myocytes cell membrane permeability caused by cardiac inflammation, in turn due to inflammatory cellular infiltration and cytokine release $^{2,3}$. Cardiac inflammation in connective tissue diseases (CTD) can manifest with a huge range of severity from transient leakage of the cytosolic component and loss of sarcolemma integrity during reversible ischemia to continuous release when ischemic or inflammatory injury is persistent and irreversible ${ }^{4}$. Similarly, NT-proBNP has been found to play a key role in the complex multiorgan and cellular adaptation in heart failure, regardless of the initial injury, and its increase could be sustained by various and complex pathogenic mechanisms ${ }^{4,5}$. As a consequence, in a complex systemic disease such as SLE, various pathogenic events could explain a persistent increase of cardiac biomarkers, and need to be comprehensively investigated. Some that have been reported are subclinical myocardial inflammation, definite myocarditis, and thrombotic microangiopathy due to antiphospholipid antibodies $4,6,7,8,9$.

In this study, the identification of increased cardiac biomarkers in 16 patients paved the way for a subsequent diagnostic investigation that led to a definite EBM-proven diagnosis of AM-induced cardiomyopathy (AMIC) in only 3 patients, while AMIC was considered "possible" in other 3 cases. Considering the other 10 patients, a specific diagnosis was made in 5 of them, including one case of SLE myocarditis, while in the 5 remaining patients both the pathological substrate and the final diagnosis remained elusive. Chiefly, considering the 9 patients with concomitant increase of HS-cTnI and NT-proBNP, a firm diagnosis of AMIC was done solely in 3 of them; investigations revealed a diagnosis of "possible AMIC" in 3 cases, lupus myocarditis in 1 , coronary artery disease in 1 , and unknown cause in 1 patient $^{1}$.

AMIC is a rare, hypertrophic, restrictive cardiomyopathy with diastolic dysfunction and a high prevalence of conduction abnormalities. To date, AMIC has been reported only in 47 patients (19 with SLE). Clinical presenting features are those of heart failure. Prognosis is poor, with about $45 \%$ mortality ${ }^{10}$.

The authors suggested that expensive and invasive investigations (such as EBM) are unjustified in asymptomatic individuals even thought a precise
AMIC diagnosis requires the detection of cytoplasm vacuolation and the virtually pathognomonic curvilinear bodies within cardiomyocytes on $\mathrm{EMB}^{10}$. Given the clinical importance and the dismal prognosis of AMIC, a conclusive detection of this rare drug-induced cardiotoxicity and a comprehensive differential diagnosis seems to be mandatory in SLE patients with high levels of HS-cTnI and/or NT-proBNP, especially in those using AM for a prolonged time.

What is more, though, even if the longterm consequences of persistently elevated cardiac enzymes in patients with SLE remain unknown, a recent study highlighted the prognostic significance of high troponin and/or NT-proBNP serum levels in patients affected by other CTD, mainly systemic sclerosis, even if asymptomatic ${ }^{4}$. A persistent elevation of troponin, particularly, seems to mirror subclinical myocardial inflammatory damage that could be revealed only by EBM. Conversely, noninvasive assessments, including cardiac magnetic resonance, could fail to detect an early-stage subclinical myocarditis. For these reasons, we strongly believe that a comprehensive diagnostic algorithm including an invasive assessment and EBM could be of greatest importance in CTD patients with increased cardiac biomarkers. A prompt and certain identification of the pathogenic substrate responsible for TnT and/or BNP increase is crucial to allow a pathogenic-driven therapeutic intervention.

\section{GIACOMO DE LUCA $@$, MD; CORRADO CAMPOCHIARO, MD;}

LORENZO DAGNA @ , MD, Professor, Unit of Immunology,

Rheumatology, Allergy and Rare Diseases, Institute for Research and Health Care San Raffaele Scientific Institute, Vita-Salute San Raffaele University, Milan, Italy. Address correspondence to Dr. G. De Luca, Unit of Immunology, Rheumatology, Allergy and Rare Diseases, IRCCS San Raffaele Scientific Institute, Vita-Salute San Raffaele University, Via Olgettina 60, 20132, Milan, Italy. E-mail: deluca.giacomo@hsr.it

\section{REFERENCES}

1. Tselios K, Gladman DD, Harvey P, Akhtari S, Su J, Urowitz MB. Abnormal cardiac biomarkers in patients with systemic lupus erythematosus and no prior heart disease: a consequence of antimalarials? J Rheumatol 2019;46:64-9.

2. Mair J, Lindahl B, Hammarsten O, Müller C, Giannitsis E, Huber K, et al. European Society of Cardiology (ESC) Study Group on Biomarkers in Cardiology of the Acute Cardiovascular Care Association (ACCA). How is cardiac troponin released from injured myocardium? Eur Heart J Acute Cardiovasc Care 2018;7:553-60.

3. Hammarsten O, Mair J, Möckel M, Lindahl B, Jaffe AS. Possible mechanisms behind cardiac troponin elevations. Biomarkers 2018;6:1-26.

4. Bosello S, De Luca G, Berardi G, Canestrari G, de Waure C, Gabrielli FA, et al. Cardiac troponin T and NT-proBNP as diagnostic and prognostic biomarkers of primary cardiac involvement and disease severity in systemic sclerosis: A prospective study. Eur J Intern Med 2019;60:46-53.

5. De Sa DD, Chen HH. The role of natriuretic peptides in heart failure. Curr Cardiol Rep 2008;10:182-9.

6. Bosello S, De Luca G, Ferraccioli G. Troponin in stable ischemic heart disease and diabetes. N Engl J Med 2015;373:1977-8.

7. Peretto G, Sala S, De Luca G, Campochiaro C, Sartorelli S, Cappelletti AM, et al. Impact of systemic immune-mediated diseases on clinical features and prognosis of patients with biopsy-proved myocarditis. Int J Cardiol 2019;280:110-16.

8. Denas G, Jose SP, Bracco A, Zoppellaro G, Pengo V. Antiphospholipid syndrome and the heart: A case series and literature review. Autoimmun Rev 2015;14:214-22.

9. De Luca G, Bosello S, Leone AM, Gabrielli F, Pelargonio G, Inzani F, et al. Life-threatening arrhythmias in a scleroderma patient: the role of myocardial inflammation in arrhythmic outburst. Scand J Rheumatol 2017;46:78-80. 
10. Tselios K, Deeb M, Gladman DD, Harvey P, Urowitz MB.

Antimalarial-induced cardiomyopathy: a systematic review of the literature. Lupus 2018;27:591-9.

First Release July 1 2019; J Rheumatol 2019;46:10;

doi:10.3899/jrheum.190078 